\title{
ICU respiratory admissions data for influenza severity surveillance?
}

\author{
Liselotte van Asten ${ }^{1}$, Angie Luna Pinzon ${ }^{1}$, Dylan W. de Lange 2, 3, Evert de Jonge 2, 4, \\ Frederika Dijkstra ${ }^{1}$, Sierk Marbus ${ }^{1}$, Gé Donker ${ }^{5}$, Wim van der Hoek ${ }^{1}$, Nicolette F. de Keizer ${ }^{2,6}$ \\ ${ }^{1}$ Centre for Infectious Disease Control Netherlands, National Institute for Public Health and the Environment (RIVM), Utrecht, Bilthoven, Netherlands, \\ ${ }^{2}$ National Intensive Care Evaluation, Amsterdam, Netherlands, ${ }^{3}$ Department of Intensive Care Medicine, University Medical Center, University Utrecht, \\ Utrecht, Netherlands, ${ }^{4}$ Department of Intensive Care, Leiden University Medical Center, Leiden, Netherlands, ${ }^{5}$ Nivel Primary Care Database - sentinel \\ practices, Utrecht, Netherlands, ${ }^{6}$ Department of Medical Informatics, Amsterdam UMC, Amsterdam Public Health research institute, Amsterdam, \\ Netherlands
}

Objective

Intensive Care Unit (ICU) data are registered for quality monitoring in the Netherlands with near 100\% coverage. They are a 'big data' type source that may be useful for infectious disease surveillance. We explored their potential to enhance the surveillance of influenza which is currently based on the milder end of the disease spectrum. We ultimately aim to set up a real-surveillance system of severe acute respiratory infections.

\section{Introduction}

While influenza-like-illness (ILI) surveillance is well-organized at primary care level in Europe, little data is available on more severe cases. With retrospective data from ICU's we aim to fill this current knowledge gap and to explore its worth for prospective surveillance. Using multiple parameters proposed by the World Health Organization we estimated the burden of severe acute respiratory infections (SARI) to ICU and how this varies between influenza epidemics.

\section{Methods}

We analyzed weekly ICU admissions of adults in the Netherlands (2007-2016) from the national intensive care evaluation (NICE) quality registry $(100 \%$ coverage of adult ICU in 2016; population size 14 million adults. A SARI syndrome was defined as admission diagnosis being any of 6 pneumonia or pulmonary sepsis codes in the Acute Physiology and Chronic Health Evaluation IV (APACHE IV) prognostic model. Influenza epidemic periods were retrieved from primary care sentinel influenza surveillance data. In recent years NICE has explored and promoted increased timeliness and automation of data transfer.

\section{Results}

Annually, 11-14\% of medical admissions to adult ICUs were for a SARI (5-25\% weekly). Admissions for bacterial pneumonia (59\%) and pulmonary sepsis (25\%) contributed most to ICU-SARI. Between influenza epidemics, severity indicators varied: ICUSARI incidence (between 558-2,400 cumulated admissions nation-wide, rate: 0.40-1.71/10,000 inhabitants), average APACHE score (between 71-78), ICU-SARI mortality (between 13-20\%), ICU-SARI/ILI ratio (between

8-17 SARI ICU cases per 1,000 expected medically attended influenza-like-illness in primary care), peak incidence (between 101188 ICU-SARI admissions nationally in the highest week, rate: between 0.07-0.13/10,000 population).

ICUs use different types of electronic health records (EHRs). Data submitted to the NICE registry is mainly based on routinely collected data extracted from these EHRs. The timeliness of data submission varies between a few weeks and three months. Together with ICUs, the NICE registry has recently undertaken actions to increase timeliness of ICU data submission.

\section{Conclusions}

In ICU data, great variation can be seen between the yearly influenza epidemic periods in terms of different influenza severity parameters. The parameters also complement each other by reflecting different aspects of severity. Prospective syndromic ICUSARI surveillance, as proposed by the World Health Organization would provide insight into severity of ongoing influenza epidemics which differ from season to season. 
Currently a subset of hospitals provide data with a 6-week delay. This can be a worthwhile addition to current influenza surveillance, which, while timelier, is based on milder cases seen by general practitioners (primary care). Future increases in data timeliness will remain an aim. 